\title{
Predicting of Sn11Sb5.5Cu babbitt fracture using computer simulation at equal-channel angular extrusion
}

\author{
A. Kh. Valeeva ${ }^{1,2,}$, A. Kh. Akhunova ${ }^{1,3}$, I. Sh. Valeev ${ }^{1,2}$ \\ †valeevs@mail.ru \\ ${ }^{1}$ Institute for Metals Superplasticity Problems of RAS, 39 Khalturin st., Ufa, 450001, Russia \\ ${ }^{2}$ Bashkir State University, 32 Zaki Validi st., Ufa, 450076, Russia \\ ${ }^{3}$ Ufa State Aviation Technical University, 12 K. Marx st., Ufa, 450000, Russia
}

\begin{abstract}
The severe plastic deformation (SPD) methods are widely used to form ultrafine-grained structure in metals and alloys. They lead to a significant change in the physical and mechanical properties of materials. Since large deformations are accumulated during SPD the material loses its ability to deform and begins to break down. The purpose of this work was to predict the fracture of $\mathrm{Sn} 11 \mathrm{Sb} 5.5 \mathrm{Cu}$ babbitt with a different microstructure at equal-channel angular pressing (ECAP) based on the finite element modeling. The tin babbit $(11 \% \mathrm{Sb}, 5.5 \% \mathrm{Cu}$, Sn the rest, weight \%), obtained by conventional casting with a different crystallization rate was chosen for study. The increase of crystallization rate led to the formation of a structure with smaller and uniformly distributed intermetallic particles. The equipment for ECAP consists of a matrix with vertical and horizontal channels crossed at angle $90^{\circ}$ and a punch located in the vertical channel. ECAP was conducted at ambient temperature, a rod of square cross-section $9.7 \times 9.7 \mathrm{~mm}$ and a length of $60.0 \mathrm{~mm}$ was deformed. The transfer speed of the punch was $1 \mathrm{~mm} / \mathrm{min}$. Computer modeling was performed using the DEFORM-2D software package. A model of the above described equipment and the sample was built. The comparison of computer simulation data and natural experiment was performed. It is shown that the metal damage calculated by the Cockroft-Latham method, installed in the DEFORM-2D, makes it possible to predict the failure of the babbitt Sn11Sb5.5Cu samples with different structures at ECAP.
\end{abstract}

Keywords: equal-channel angular pressing (ECAP), computer simulation, babbitt $\mathrm{Sn} 11 \mathrm{Sb} 5.5 \mathrm{Cu}$, damage.

УДК: $621.777: 539.538$

\section{Прогнозирование разрушения баббита Б83 при равноканальном угловом прессовании методом компьютерного моделирования}

\author{
Валеева А. Х. ${ }^{1,2, \dagger}$, Ахунова А. Х. ${ }^{1,3}$, Валеев И.Ш. ${ }^{1,2}$ \\ ${ }^{1}$ Институт проблем сверхпластичности металлов РАН, ул. Ст.Халтурина, 39, Уфа, 450001, Россия \\ ${ }^{2}$ Башкирский государственный университет, ул. Заки Валиди, 32, Уфа, 450076, Россия \\ 3Уфимский государственный авиационный технический университет, ул. К. Маркса, 12, Уфа, 450000, Россия
}

Для формирования ультрамелкозернистой структуры в металлах и сплавах используются методы интенсивной пластической деформации (ИПД), приводящие к существенному изменению физических и механических свойств материалов. Поскольку накапливаемые в ходе такой обработки деформации велики, материал заготовки начинает разрушаться. Цель данной работы заключается в прогнозировании разрушения при равноканальном угловом прессовании (РКУП) заготовки из баббита Б83 с различной микроструктурой на основании результатов конечно-элементного моделирования. В качестве материала исследования был использован баббит Б83 (11\% Sb, 5,5\% Cu, остальное $\mathrm{Sn}$, вес.\%), полученный обычным литьем в кокиль при различной скорости кристаллизации. Изменение скорости кристаллизации позволяло получить микроструктуры с различными размерами интерметаллидных фаз. Оснастка для РКУП состояла из матрицы с вертикальным и горизонтальным каналами, пересекающимися под прямым углом, и пуансона, находящегося в вертикальном канале матрицы. РКУП осуществляли при комнатной температуре, деформировали пруток квадратного поперечного сечения 9,7×9,7 мм и длиной 60,0 мм. Скорость перемещения пуансона составляла 1 мм/мин. Компьютерное моделирование выполняли в двухмерной постановке с помощью пакета прикладных программ DEFORM-2D. Строили модель вышеописанной оснастки и образца. Проводили сравнение данных компьютерного моделирования и натурного эксперимента. Результаты исследования показали, что методика расчета поврежденности металла Кокрофта-Лэтэма, установленная в указанном программном комплексе, позволяет с достаточной точностью прогнозировать характер разрушения образцов из баббита Б83 с различной структурой при РКУП.

Ключевые слова: равноканальное угловое прессование (РКУП), компьютерное моделирование, баббит Б83, поврежденность. 


\section{1. Введение}

Методы интенсивной пластической деформации (ИПД) используются для формирования ультрамелкозернистой структуры в металлах и сплавах, обеспечивая существенное изменение их физических и механических свойств [1-12]. Измельчение при равноканальном угловом прессовании (РКУП) и кручении под гидростатическим давлением (КГД) крупных интерметаллидных частиц, присутствующих в баббитах, оказывает положительное влияние на усталостную прочность и сопротивление хрупкому разрушению, обеспечивает существенное снижение коэффициента граничного трения [13-15.]

Важным вопросом при разработке процессов ИПД является прогнозирование разрушения металла. Накапливаемые при такой обработке деформации велики, поэтому материал заготовки, исчерпав свою способность к деформированию, после, например, определенного числа проходов РКУП, начинает разрушаться. Предельные деформации ограничиваются вязким разрушением материала, сопровождающимся развитием и увеличением количества микропор и микротрещин. [17].

Данная работа посвящена прогнозированию разрушения при РКУП заготовки из баббита Б83 с различной микроструктурой на основании результатов конечноэлементного моделирования в среде программного продукта DEFORM-2D.

\section{2. Материалы и методики}

В качестве материала исследования был выбран баббит Б83 (11\% Sb, 5,5\% Cu, остальное - Sn, вес.\%), полученный обычным литьем в кокиль. Микроструктура баббита варьировалась скоростью охлаждения при кристаллизации [18]. Оснастка для РКУП состояла из матрицы с вертикальным и горизонтальным каналами, пересекающимися под прямым углом, и пуансона, находящегося в вертикальном канале матрицы. РКУП проводили при комнатной температуре, деформировали пруток квадратного поперечного сечения площадью $9,7 \times 9,7$ мм и длиной 60,0 мм. Скорость перемещения пуансона составляла $1 \mathrm{mм/мин.}$

Компьютерное моделирование выполнялось в двухмерной постановке с помощью пакета прикладных программ DEFORM-2D. Строили модель вышеописанной оснастки и образца (Рис. 1). Пуансону и матрице задавались свойства абсолютно жесткого тела. Материал прутка принимался пластичным, и описывался экспериментальными кривыми, полученными при сжатии образцов из баббита Б83 при комнатной температуре [18]. Контактные условия на границах «пруток - матрица» и «пруток - пуансон» описывались моделью трения по Зибелю [19]. Величина коэффициента трения принималась равной 0.03. Деформируемое тело разбивали на двадцатиузловые изопараметрические конечные элементы с квадратичной аппроксимацией поля перемещений. Количество элементов - 5200. Моделировали один проход пуансона, без противодавления.
При моделировании РКУП для оценки поврежденности металла была использована модель разрушения Кокрофта-Лэтэма $[17,20-23]$, установленная в программном комплексе DEFORM-2D. Критерий Кокрофта-Лэтэма является показателем предельной деформируемости материала и основан на оценке полной работы деформации на единицу объема в точке разрушения. Согласно критерию, показатель поврежденности металла вычисляется по формуле:

$$
D=\int_{0}^{\varepsilon_{i}} \frac{\sigma_{1}}{\sigma_{i}} d \varepsilon_{i},
$$

где $\sigma_{1}-$ максимальное главное напряжение, $\sigma_{i}-$ интенсивность напряжений, $\varepsilon_{i}-$ интенсивность деформации.

\section{3. Результаты и обсуждения}

Микроструктура баббита Б83 в литом состоянии при различной скорости охлаждении во время кристаллизации представлена на Рис. 2.

На фотографиях, полученных оптической металлографией, видно, что баббит, полученный при обычном охлаждении, характеризуется микроструктурой с крупными кубическими частицами $\beta$-фазы SnSb размером

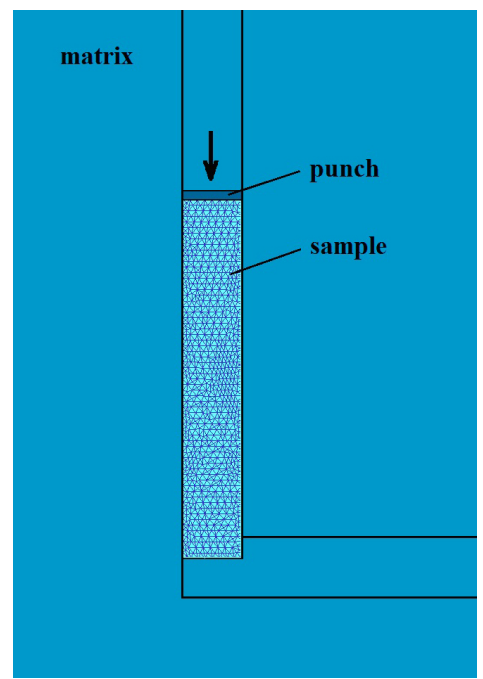

Рис. 1. Конечно-элементная модель оснастки и образца для РКУП.

Fig. 1. Finite-element model of the matrix and sample at ECAP.

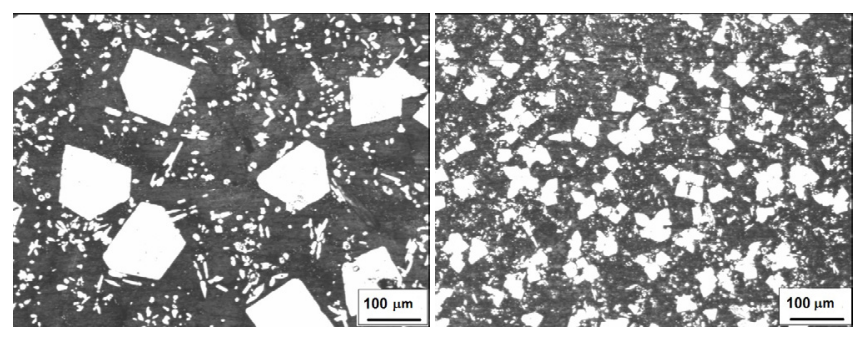

a

b

Рис. 2. Микроструктура баббита Б83 при кристаллизации: с обычной скоростью (a) и с повышенной скоростью (b).

Fig. 2. Microstructure of babbit $\mathrm{Sn} 11 \% \mathrm{Sb} 5.5 \% \mathrm{Cu}$ at crystallization with: the usual cooling rate (a) and increased rate (b). 
100-200 мкм и п-фазой в виде крупных звездочек игл соединения $\mathrm{Cu}_{6} \mathrm{Sn}_{5}$, неравномерно распределенными в матричной а-фазе (Рис. 2a). Повышение скорости кристаллизации при охлаждении водой приводит к уменьшению размера $\beta$-фазы на порядок, кроме того иглы $\eta$-фазы не успевают вырасти, оставаясь мелкими глобулярными частицами. Обе интерметаллидные фазы равномерно распределены в матрице (Рис. 2b). При этом происходит соответствующее изменение механических свойств [18,24,25], выражающееся в повышении напряжений течения с уменьшением размера частиц $\beta$-фазы.

На Рис. 3 представлены качественные картины распределения напряжений и деформаций в очаге деформации прутка из баббита Б83, полученного при обычном охлаждении во время кристаллизации. Можно видеть, что при переходе прутка из вертикального канала в горизонтальный, образуется очаг деформации, в верхней зоне которого формируются максимальные напряжения (Рис. 3a). Неравномерное распределение напряжений и затрудненность движения металла по всей нижней поверхности горизонтального канала, вследствие наличия трения, приводит к неравномерному течению материала и к образованию застойных зон деформации (Рис. 3b). При таких условиях поверхностные слои прутка испытывают сильное растяжение. Кроме того, скорости течения материала верхней и нижней поверхности прутка сильно различаются. Сочетание всех этих факторов в итоге вызывает гребнеобразное изменение формы прутка.

На Рис. 4 представлены эпюры значений критерия разрушения $D$, полученные в результате компьютерного моделирования. Видно, что большему разрушению подвержена область контакта верхней поверхности прутка с матрицей. Значения $D$ в данной области почти в три раза выше, чем в остальном объеме металла. Это объясняется наличием трения на контактной поверхности прутка с нижней поверхностью выходного канала. Трение в углу пересечения каналов является основным отрицательным фактором, влияющим на целостность образца. В образце, которому заданы свойства материала, полученного при скоростной кристаллизации, происходит образование 5 гребней, при этом минимальная глубина составляет 1,98 мм, а максимальная - 3,37 мм. В образце, которому заданы свойства материала, полученного при кристаллизации с повышенной скоростью охлаждения, сопровождающемся уменьшением размера частиц интерметаллидных фаз, образуется 4 гребня, но при этом глубина гребней достигает 3,95 мм и площадь областей, характеризующихся повышенными значениями критерия разрушения на них, увеличивается (Рис. 4b).

Внешний вид заготовок из баббита Б83 с различной микроструктурой после натурного эксперимента по равноканальному угловому прессованию представлен на Рис. 5.

Полученные результаты качественно совпадают с прогнозом компьютерного моделирования. Предсказанное гребнеобразное изменение формы, в натурном эксперименте вылилось в образование трещин на верх-

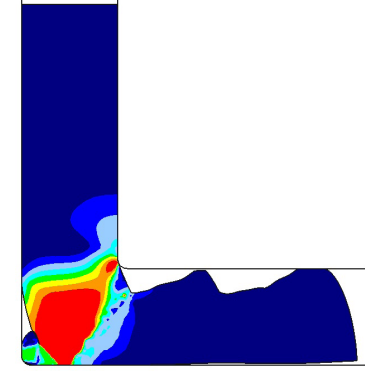

a

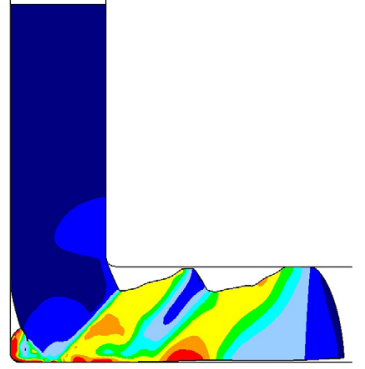

b
Pис. 3. (Color online) Качественная картина распределения интенсивности напряжений (a) и деформаций по ван Мизесу (b) в очаге деформации в прутке из баббита Б83, полученного при кристаллизации с обычной скоростью охлаждения.

Fig. 3. (Color online) A qualitative picture of the intensity of stresses (a) and van Mises deformations (b) in the deformation center in a bar of Babbit $\mathrm{Sn} 11 \mathrm{Sb} 5.5 \mathrm{Cu}$, obtained by crystallization with the usual cooling rate.
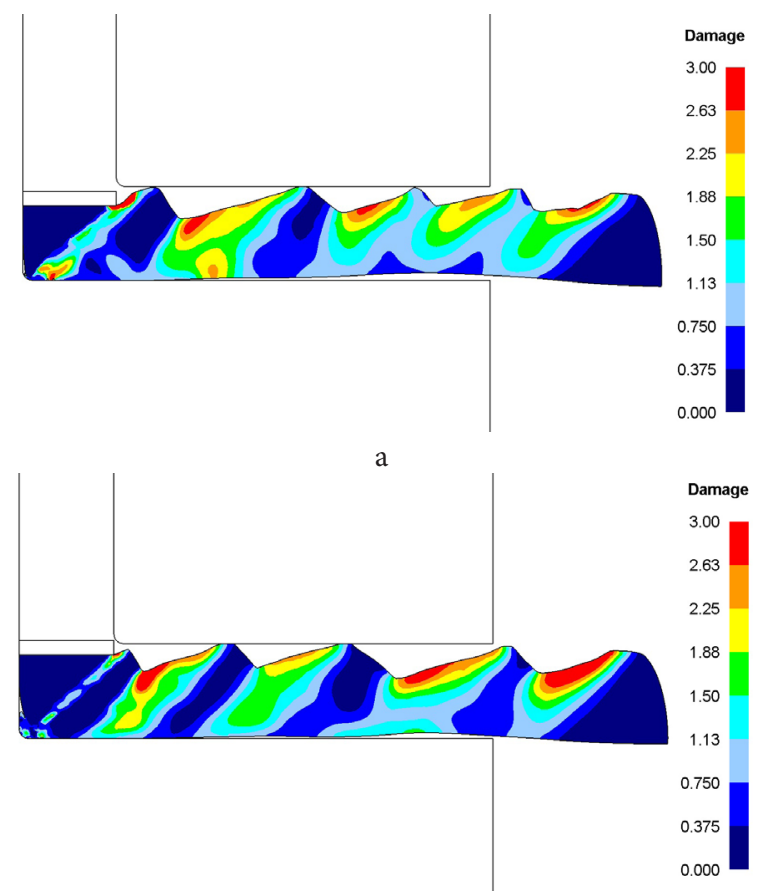

Pис. 4. (Color online) Эпюры значений критерия разрушения $D$ в прутке из баббита Б83, полученном при кристаллизации: с обычной скоростью охлаждения (а) и повышенной скоростью (b).

Fig.4. (Color online) The Damage diagrams in Sn11Sb5.5Cu obtained at crystallization: with the usual cooling rate (a) and increased rate (b).

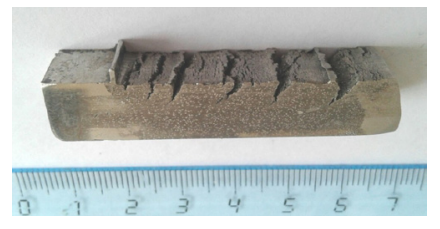

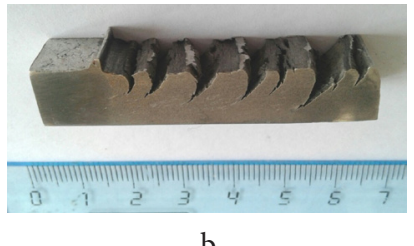

b
Рис. 5. Вид заготовок из баббита Б83, полученных при: обычной скорости охлаждения при кристаллизации (а) и повышенной скорости (b) после РКУП.

Fig. 5. The type of $S n 11 \%$ Sb5.55Cu rods obtained at the usual cooling rate during crystallization (a) and increased rate (b) after ECAP. 
ней поверхности прутка (Рис. 5). В заготовках, полученных при обычной скорости охлаждения, с крупными частицами интерметаллидных фаз образуется большее число мелких трещин небольшой глубины (Рис. 5a), в то время как в заготовках, полученных при скоростном охлаждении, с мелкими частицами, наблюдается образование меньшего числа глубоких трещин (Рис. 5b), как и в компьютерной модели.

\section{4. Вывод}

Использование при компьютерном моделировании процесса равноканального углового прессования модели разрушения Кокрофта-Лэтэма, установленой в программном комплексе DEFORM-2D, позволяет с достаточной точностью прогнозировать качественный характер разрушения образцов из баббита Б83 с различной микроструктурой.

Благодарности/Acknowledgements. Работа выполнена в рамках государственного задания ИПСМ РАН (Рег. No AAAA-A17-117041310218-5)./The present work was accomplished according to the state assignment of IMSP RAS (Reg.No AAAA-A17-117041310218-5).

\section{Литература/References}

1. N. I. Noskova, R. R. Muluykov. Submicrocrystalline and nanocrystalline metalls and alloys. Yekaterinburg, Urals Branch of the Russian Academy of Sciences (2003) 279 р. (in Russian). [Н.И. Носкова, Р. Р. Мулюков. Субмикрокристаллические и нанокристаллические металлы и сплавы. Екатеринбург, Уральское отделение РАН (2003) 279 с.]

2. A.P. Zhilyaev, A.I. Pshenichnyuk. Superplasticity and grain boundaries in ultrafine materials. Moscow, FIZMATLIT (2008) 320 p. (in Russian) [А.П. Жиляев, А.И. Пшеничнюк. Сверхпластичность и границы зерен в ультрамелкозернистых материалах. Москва, ФИЗМАТЛИТ (2008) 320 с.]

3. A.P. Zhilyaev, T.G. Langdon. Prog. Mat. Sci. 53, 893 (2008). DOI: 10.1016/j.pmatsci.2008.03.002

4. E. G. Astafurova, G.G. Zakharova, E. V. Naydenkin, G. I. Raab, P.D. Odessky, S.V. Dobatkin. Letters on materials. 1(4), 198 (2011). (in Russian) [Е. Г. Астафурова, Г. Г.Захарова,Е. В.Найденкин,Г.И.Рааб,П.Д.Одесский, С. В. Добаткин. Письма о материалах. 1(4), 198 (2011).] DOI: 10.22226/2410-3535-2011-4-198-202

5. Yuechen Dun, V.D. Sitdikov, I. V. Aleksandrov, J. T. Vang. Letters on materials. 3(2), 169 (2013) (in Rusian). [Юечэн Дун, Ситдиков В.Д., Александров И.В., Ванг Дж. Т. Письма о материалах. 3(2), 169 (2013).] DOI: $10.22226 / 2410-3535-2013-2-169-172$

6. E. V. Avtokratova, O. E. Mukhametdinova, O. Sh. Sitdikov, M.V. Markushev, S. V. S. N. Murty, M. J. N. V. Prasad, B.P. Kashyap. Letters on materials. 4(2), 93 (2014). DOI: 10.22226/2410-3535-2014-2-93-95

7. S. Sabbaghianrad, T. G. Langdon. Letters on materials. 5(3), 335 (2015). DOI: 10.22226/2410-3535-2015-3-335-340

8. M. Kawasaki, R.B. Figueiredo, T.G. Langdon.
Letters on materials. 5(3), 233

(2015).

DOI: 10.22226/2410-3535-2015-3-233-239

9. E. Avtokratova, O. Sitdikov, M. Markushev. Letters on materials. 5(3), 319 (2015). DOI: $10.22226 / 2410-3535-2015-3-319-323$

10. E. I. Fakhretdinova, E.V. Bobruk, G.Yu. Sagitova, G. I. Raab. Letters on materials. 5(2), 202 (2015). (in Rusian) [Э.И. Фахретдинова, Е.В. Бобрук, Г. Ю. Сагитова, Г.И. Рааб. Письма о материалах 5(2), 202 (2015).] DOI: 10.22226/2410-3535-2015-2-202-206

11. M. Demirtas, G. Purcek, H. Yanar, Z. J. Zhang, Z.F. Zhang. Letters on materials. 5(3), 328 (2015). DOI: $10.22226 / 2410-3535-2015-3-328-334$

12. G. R. Khalikova, G. F. Korznikova, V. G. Trifonov. Letters on materials. 7(1), 3 (2017). (in Russian) [Г. Р. Халикова, Г. Ф. Корзникова, В.Г. Трифонов. Письма о материалах. 7(1), 3 (2017).] DOI: 10.22226/2410-3535-2017-1-3-7

13. A.Kh. Valeeva, I.Sh. Valeev, R.R. Mulyukov, R. Khisamov. Letters on materials. 6(4), 347 (2016). (in Russian) [А.Х. Валеева, И.Ш. Валеев, Р. Р. Мулюков, P.Х. Хисамов. Письма о материалах. 6(4), 347 (2016).] DOI: 10.22226/2410-3535-2016-4-347-349

14. L.G. Korshunov, N.I. Noskova, A.V. Korznikov, N. L. Chernenko, N.F. Vil'danova. The Physics of Metals and Metallography. 108, 519 (2009).

15. N. I. Noskova, L. G. Korshunov, A. V. Korznikov. Metal Science and Heat Treatment. 50, 593 (2008).

16. A. Kh. Akhunova, A. Kh. Valeeva, I.Sh. Valeev. BPMS 14(3), 322 (2017). (in Russian) [Ахунова A.X., Валеева А. Х., Валеев И.Ш. ФПСМ. 14(3), 322 (2017).]

17. A. V. Botkin, R.Z. Valiev, S. V. Dubinina, G.I. Raab, P.S. Stepin. Vestnik of NMSTU. 4, 38 (2011). (in Russian) [Боткин А.В., Валиев Р.3., Дубинина С.В., Рааб Г.И., Степин П.С. Вестник МГТУ им. Г.И. Носова. 4, 38 (2011).]

18. I. Sh. Valeev, A. Kh. Valeeva, A. Kh. Akhunova. Letters on Materials. 7(3), 292 (2017). (in Russian) [Валеев И.Ш., Валеева А. Х., Ахунова А. Х. Письма о материалах. 7(3), 292 (2017).] DOI: 10.22226/2410-3535-2017-3-292-295

19. V.L. Kolmogorov. Mechanics of metal working with pressure: a textbook for high schools. $2^{\text {nd }}$ ed. Ekaterinburg, Publishing House of the USTU-UPI (2001) 836 p. (in Russian) [Колмогоров В.Л. Механика обработки металлов давлением. Екатеринбург, УГТУ - УПИ (2001) 836 c.] ISBN 5-321-00050-6

20. M. G. Cockcroft, D. J. Latham. J. Inst.Metals. 96, 33 (1968). 21. R. Hambli, M. Reszka. Int. J. Mech. Sci. 44, 1349 (2002).

22. N. Ogawa, M. Shiomi, K. Osakada. Int. J. Mach. Tools Manuf. 42, 607 (2002).

23. A.V. Botkin, R.Z. Valiev, P.S. Stepin, A.Kh. Baimukhametov. Deformation and destruction of materials. 7, 17 (2011). (in Russian) [Боткин А. B., Валиев Р.3., Степин П.С., Баймухаметов А.Х. Деформация и разрушение материалов. 7, 17 (2011).]

24. F. A. Sadykov, N.P. Barykin, I.Sh. Valeev. Physics and chemistry of materials treatment. 2, 86 (2001). (in Russian) [Садыков Ф.А., Барыкин Н.П., Валеев И.Ш. Физика и химия обработки материалов. 2, 86 (2001).]

25. F. A. Sadykov, N.P. Barykin, I. Sh. Valeev. Strength of Materials. 34(2), 196 (2002). 